\title{
Formation of Etch Beaks on the (111) Surfaces of Rapidly-Cooled Copper Crystals*
}

\author{
By Shigeo Sugawara** and Jirô Watanabé**
}

\begin{abstract}
The (111) surfaces of copper crystals cooled rapidly from $1273 \mathrm{~K}$ in a purified argon atmosphere were etched with three kinds of dislocation etchants, and the etch patterns were studied by means of optical and electron microscopies. From the replica examinations with an optical microscope dark and deep etch pits with beaks were found in addition to normal dislocation etch pits. The density of the former pits amounted to $10^{7}-10^{8} \mathrm{~m}^{-2}$, two orders in magnitude less than that of the latter ones. Detailed observations of the replicas with an electron microscope revealed that the beaks extended into the crystal from the base of the etch pits along an oblique $\langle 110\rangle$ direction, though details of their shapes varied with the etching solutions. When the crystal was etched for a long period, the beaked etch pits gradually changed from sharply-pointed and deep ones to flatbottomed and shallow ones, and finally disappeared, while small beaked ones newly appeared at some other places. It is noted that such etch pits could not be observed in slowly-cooled copper crystals. Defect ribbons with a somewhat complicated structure were observed in thin films obtained from the rapidly-cooled copper crystals, which might presumably be associated with the etch beaks.
\end{abstract}

(Received February 4, 1982)

\section{Introduction}

When a crystal is etched under appropriate thermal or chemical conditions, etch pits are formed on a low index crystal surface. The mature etch pits are composed of facets or edges developed by etching, which arrange corresponding to a crystal symmetry of the crystal surface. Accordingly, the shape of the etch pits reflects also the crystal symmetry concerned. However, it was early found that there were anomalies in an actual development of etch pits masking their symmetry. Asymmetric etch pits are called "anomalous etch pits". Etch beaks are thin tunnels which extend into the interior of the crystal from the base of the etch $\mathrm{pit}^{(1)-(3)}$, and when the extending direction is at random, the beaked pits are said to be of the anomalous type.

In 1958, Lovell ${ }^{(4)}$ reported first that beaked pits were nucleated at the sites of lattice defects in apatite crystals. She observed two kinds of beaks on the (0001) surface of apatite crystals etched with tartaric or citric acid (both at 333-

* This paper was originally published in Japanese in J. Japan Inst. Metals, 45 (1981), 996.

** Department of Metallurgy, Mining College, Akita University, Akita 010, Japan.
$343 \mathrm{~K}$ ); one penetrated perpendicularly from the bottom of hexagonal etch pits and the other extended along several oblique directions. The former type was found to correspond with an edge dislocation, since they arranged into a low-angle tilt boundary. On the other hand, it was shown by Fleischer et al. ${ }^{(5)}$ in 1964 that the latter type resulted from a preferential chemical attack of the tracks of spontaneous fissionfragmentation of uranium impurities occurring over a geological time. Such beaks were revealed on the (0001) surface of apatite and on the cleavage plane of muscovite mica crystals both of which had been exposed to a laboratory source of fission fragments ${ }^{(5)}$ as well as on the (1010) surface of natural apatite crystals ${ }^{(6)}$. Another anomalous phenomenon is development of etch pits with wings. The wings are wedges which extend sideways from the sides of etch pits. Patel and Raju( ${ }^{(7)}$ found that the tracks of fission fragments were responsible for winged pits revealed on the cleavage plane of selenite crystals.

It must be added that evidences for beaked pits to correspond to dislocations were obtained in some crystals other than apatite. In lithium fluoride crystals etched with an aqueous solution of fatty acids, beaked pits were nucleated at the sites of screw dislocations ${ }^{(8)(9)}$. On the other hand, in sodium chloride crystals etched 
with methanol containing cadmium chloride and sodium chloride, beaked etch pits were always associated with aged edge dislocations, and its formation was found to be predominant at higher concentrations of the poison, cadmium chloride, as well as at low undersaturations of the etchant ${ }^{(10)}$. Helical dislocations were also responsible for etch beaks in quartz crystals etched with concentrated hydrofluoric $\operatorname{acid}^{(11)}$. Moreover, it was pointed out that etch pits in silicon crystals resulting from a preferential attack along the curved dislocation lines would certainly appear to have beaks if they were viewed normal to the etched surface with transmitted light ${ }^{(3)}$.

This paper reports the nature of beaked etch pits formed on the (111) surfaces of copper crystals as a result of quenching from a high temperature.

\section{Experimental Procedure}

Re-electrolyzed copper metal $(99.998 \%$ in purity) was used as a starting material. Single crystals of cylindrical rod $8-10 \mathrm{~mm}$ in diameter were grown with the [111] orientation in a purified argon atmosphere by a Czochralski method. After the crystals were orientated by a light figure method ${ }^{(12)}$, they were cut into pieces about $10 \mathrm{~mm}$ in length by an acid cutter so as to expose the (111) plane which lay perpendicular to the growth direction. Crystal specimens thus obtained were placed in a high-purity graphite boat, annealed in a horizontal furnace at $1273 \mathrm{~K}$ for $7.2 \times 10^{3} \mathrm{~s}(2 \mathrm{~h})$ under a stream of purified argon $\left(\mathrm{O}_{2}\right.$ and $\mathrm{N}_{2}<0.1 \mathrm{ppm}$, dew point $<200 \mathrm{~K}$ ) and then rapidly cooled in the furnace tube by pulling quickly to a position (temperature $\sim 320 \mathrm{~K}$ ) $19 \mathrm{~cm}$ distant from the heater. Some crystal specimens were annealed under the same condition but slowly cooled to room temperature at a rate of $7 \times 10^{-2} \mathrm{~K} \cdot \mathrm{s}^{-1}$.

The crystal specimens prepared were embedded in a copper holder with paraffine, and the (111) surface was carefully polished by sliding on a woolen cloth wetted with concentrated hydrochloric acid saturated with cupric chloride. When the surface became smooth, the crystal was removed from the holder, cleaned with organic solvents and then polished electrolytically in concentrated orthophosphoric acid at a voltage of $1.5 \mathrm{~V}$ so as to reveal a mirror like surface. The (111) surface thus treated was kept at $285 \mathrm{~K}$ by dipping into thermostated water bath for about $1.2 \times 10^{2} \mathrm{~s}$ ( $2 \mathrm{~min}$ ) and etched for 5-30 s with a dislocation etchant which was maintained at the same temperature. The etchants tested were (1) Young's etchant ${ }^{(13)}\left(1 \mathrm{kmol} / \mathrm{m}^{3}\left(\mathrm{NH}_{4}\right)_{2} \mathrm{~S}_{2} \mathrm{O}_{8}\right.$, $6 \mathrm{kmol} / \mathrm{m}^{3} \mathrm{NH}_{4} \mathrm{OH}, 0.3 \mathrm{kmol} / \mathrm{m}^{3} \mathrm{NH}_{4} \mathrm{Br}$ ), (2) Marukawa's one ${ }^{(14)} \quad\left(\mathrm{FeCl}_{3} \cdot 6 \mathrm{H}_{2} \mathrm{O} 50 \mathrm{~g}+\right.$ $\left.\mathrm{H}_{2} \mathrm{O} 103 \mathrm{~cm}^{3}+\mathrm{HCl} 80 \mathrm{~cm}^{3}+\mathrm{HBr} 3 \mathrm{~cm}^{3}\right)$ and (3) Livingston's one ${ }^{(15)} \quad\left(\mathrm{HCl} 25 \mathrm{~cm}^{3}+\right.$ $\left.\mathrm{CH}_{3} \mathrm{COOH} 15 \mathrm{~cm}^{3}+\mathrm{H}_{2} \mathrm{O} 90 \mathrm{~cm}^{3}+\mathrm{Br}_{2} 1 \mathrm{~cm}^{3}\right)$.

Optical microscopic observations of etch pits were made on the as-etched surface and on its replica film. The replica was prepared by the following technique. A plastic film (Bioden tape) wetted with solvent was affixed to the etched surface and the solvent allowed to evaporate. The resulting film was stripped directly and then a thin film of silver was evaporated onto the replica surface at an incident angle of $0.105-0.140 \mathrm{rad}\left(6^{\circ}-8^{\circ}\right)$. In order to examine the fine structure of the etch pits, electron microscopic observations were also made by the use of $\mathrm{Pt}-20 \% \mathrm{Pd}$ shadow-carbon replica.

\section{Experimental Results}

\section{Etching with Young's etchant ${ }^{(13)}$}

Photograph 1 shows replica optical micrographs of etch pits formed on the (111) surfaces of rapidly-cooled and slowly-cooled copper crystals which were etched with Young's etchant for $5 \mathrm{~s}$. Triangular pits, of which contrasts are dark, light and mixed, are found on both crystals. In addition, in the rapidlycooled crystal, anomalous dark pits with a long trail can be seen as encircled in Photo. 1(a), while such etch pits can never be found in the slowly-cooled crystal. The density of the anomalous dark pits amounted to $10^{7}-10^{8} \mathrm{~m}^{-2}$, two orders in magnitude less than the sum of normal pits with dark and light contrasts. Electron micrographs shown in Photo. 2 disclose more clearly the appearance of the anomalous pit. In Photo. 2(a), two etch pits, one 


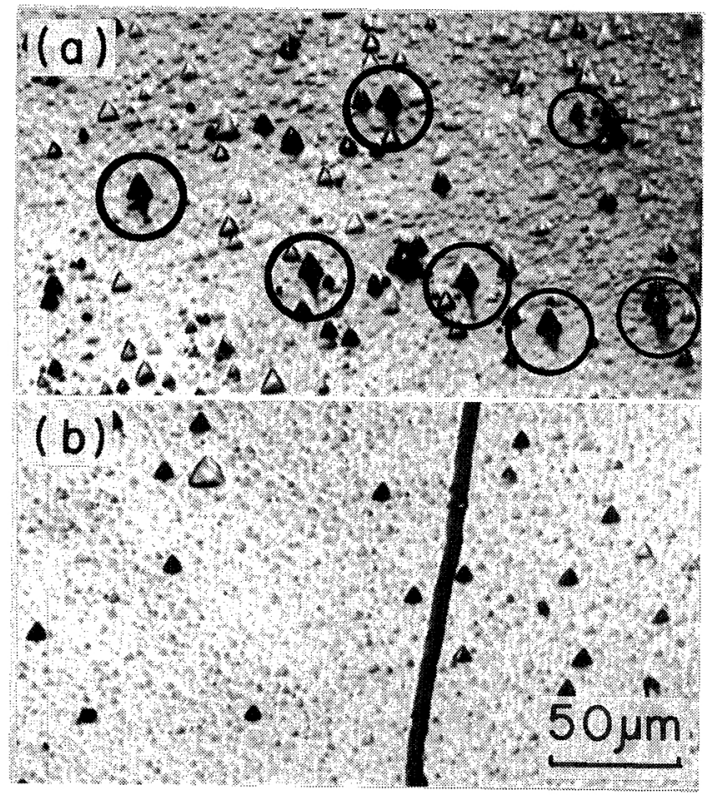

Photo. 1 Replica optical micrographs of etch pits formed on the (111) surfaces of copper crystals after etched with Young's etchant ${ }^{(13)}$ for $5 \mathrm{~s}$. (a) Rapidly-cooled from 1273 K, (b) Slowly-cooled.

corresponding to the normal one and the other to the anomalous one, are seen. From the white shadow length for the normal pit, its side slope was estimated to be about $0.23 \mathrm{rad}\left(13^{\circ}\right)$, which corresponded to the etch pit appearing as dark in the optical microscopy ${ }^{(16)}$. It is to be noticed that the etch pit appearing as light in the optical microscopy was shallower and its side slope was mostly less than $0.10 \mathrm{rad}\left(6^{\circ}\right)^{(16)}$. On the other hand, the anomalous pit is far deeper than the normal dark one and it contains some marked features at the bottom portion. The side surface of the anomalous pit was remarkably curved and its slope increased from $\sim 0.31 \mathrm{rad}\left(\sim 18^{\circ}\right)$ at the upper portion to $\sim 0.70 \mathrm{rad}\left(\sim 40^{\circ}\right)$ at the bottom portion. Moreover, when the anomalous etch pit is examined at a higher magnification, a dark band can be seen across the center of etch pit (Photo. 2(b)). This is an image of tubular tunnel, or etch beak, which extends into the crystal along an oblique direction to the (111) face. Photograph 2(c) is a view nearly along the beak, which was taken by tilting the etch pit with a tilting device. It can be seen that the beak is cylindrical in cross section (about $0.1 \mu \mathrm{m}$ in diameter) and it protrudes from an apex of square pyramid. The angle of tilt for Photo. 2(c) was $\pi / 6\left(30^{\circ}\right)$ as set at a permissible limit for the goniometer used, corresponding nearly to $[111] /[110]=\pi / 5.1\left(35^{\circ} 16^{\prime}\right)$. Hence, it is predicted that the beak may extend into the crystal along the [110] direction. It can be pointed after studying a large number of etch

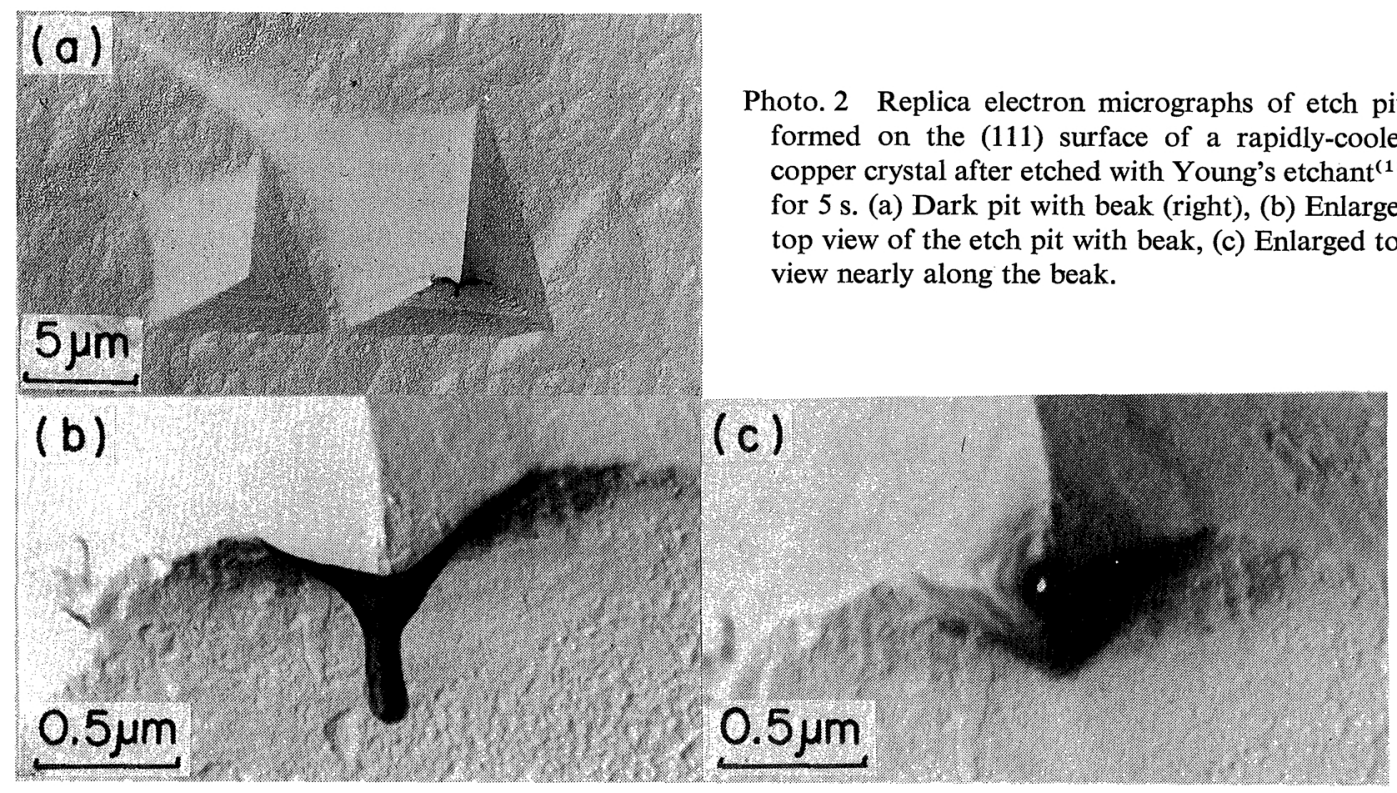




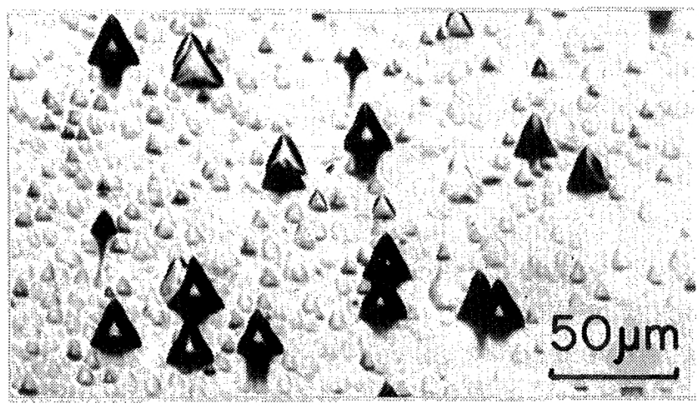

Photo. 3 Replica optical micrograph of etch pits formed on the (111) surface of a rapidly-cooled copper crystal after etched with Young's etchant ${ }^{(13)}$ for $20 \mathrm{~s}$.

pits that the beaks may always be situated along one of three oblique directions [110], [101] and [011].

Photograph 3 shows an optical micrograph of the (111) replica surface of the rapidlycooled crystal etched with Young's etchant for $20 \mathrm{~s}$. There are three kinds of anomalous etch pits in the photograph; small ones, large ones and large flat-bottomed ones. From a comparison with Photo. 1(a), it is evident that with a continuation of etching beaked etch pits grow gradually into large flat-bottomed ones and finally disappeared, while small ones are nucleated at some other places. Such a shape change could be confirmed by the successive

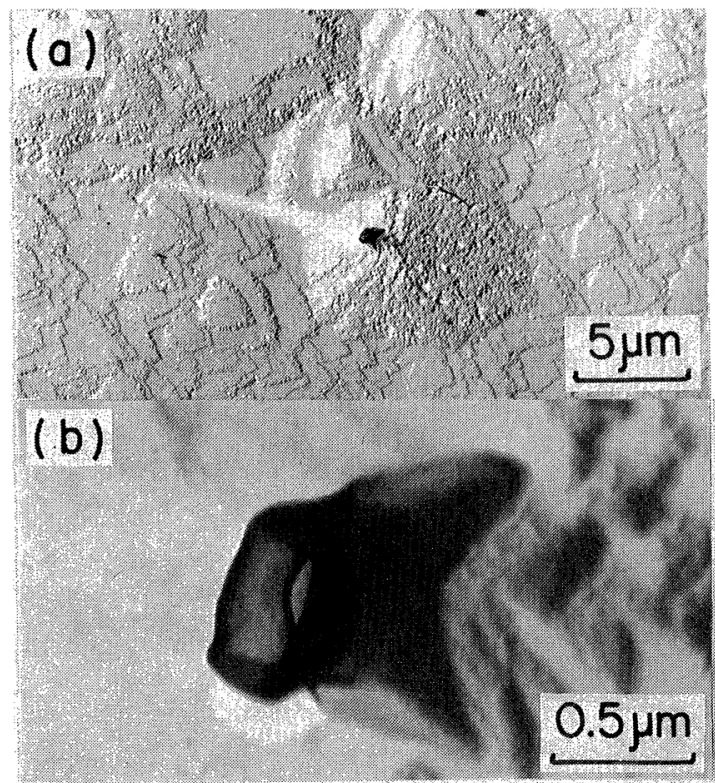

observations at $5 \mathrm{~s}$-etching intervals. These results indicate that the beaked pits may originate from a lattice defect of a relatively short length which extends into the crystal along a $\langle 110\rangle$ direction. Previously, the authors have shown that normal etch pits with dark and light contrasts revealed by Young's etchant correspond to edge dislocations of negative and positive signs, respectively. Therefore, it is concluded that the edge dislocations are not responsible for the anomalous pits.

\section{Etching with Marukawa's etchant ${ }^{(14)}$}

Photograph 4 shows a typical optical micro-

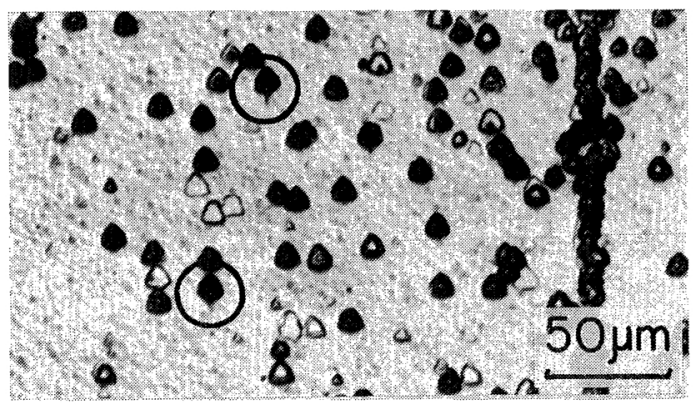

Photo. 4 Replica optical micrograph of etch pits formed on the (111) surface of a rapidly-cooled copper crystal after etched with Marukawa's etchant ${ }^{(14)}$ for $7 \mathrm{~s}$.

Photo. 5 Replica electron micrographs of an etch pit with beak formed on the (111) surface of a rapidly-cooled copper crystal after etched with Marukawa's etchant ${ }^{(14)}$ for $7 \mathrm{~s}$. (a) Dark pit with beak, (b) Enlarged top view of the etch pit with beak, (c) Enlarged top view nearly along the beak.

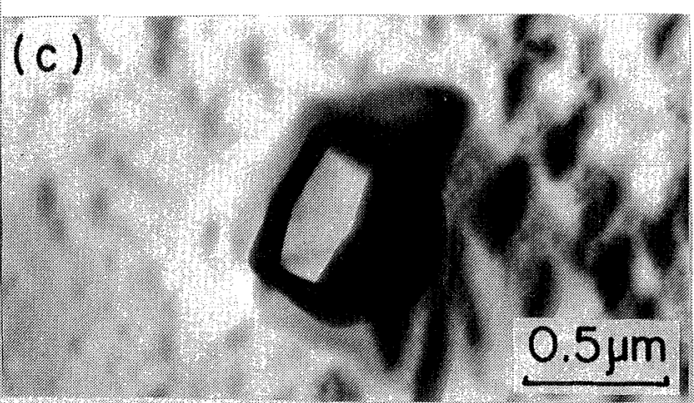


graph of a (111) replica of the rapidly-cooled copper crystal which was etched with Marukawa's etchant for $7 \mathrm{~s}$. Anomalous dark pits (encircled in the photograph) are seen in addition to normal dark, light and mixed pits. After the etched specimen was electrolytically polished lightly and re-etched with Young's etchant for $5 \mathrm{~s}$, the replica was again examined with an optical microscope. It was found that there was certainly one-to-one correspondence between the anomalous pits revealed by both etchants. Photograph 5 shows the replica electron micrographs of the anomalous pit revealed by etching with Marukawa's etchant for $7 \mathrm{~s}$. The etch pit is shallow and slightly rounded (see Photo. 5(a)). Its side slope was estimated to be $\sim 0.14 \mathrm{rad}\left(\sim 8^{\circ}\right)$, which was nearly equal to that of a normal dark pit. The beak extending from the bottom of the etch pit can be more clearly seen from the enlarged views in Photo. 5(b) and (c). (b) is the normal view, but (c) is taken by tilting (b) by an angle of $\pi / 6\left(30^{\circ}\right)$. It can be predicted here again that the beak may extend along the oblique [110] direction. A comparison of Photo. 5 with Photo. 2 shows that the shape of beak revealed by etching with Marukawa's etchant is different from that with Young's etchant, depending upon the nature of etch facets development. Marukawa's etchant

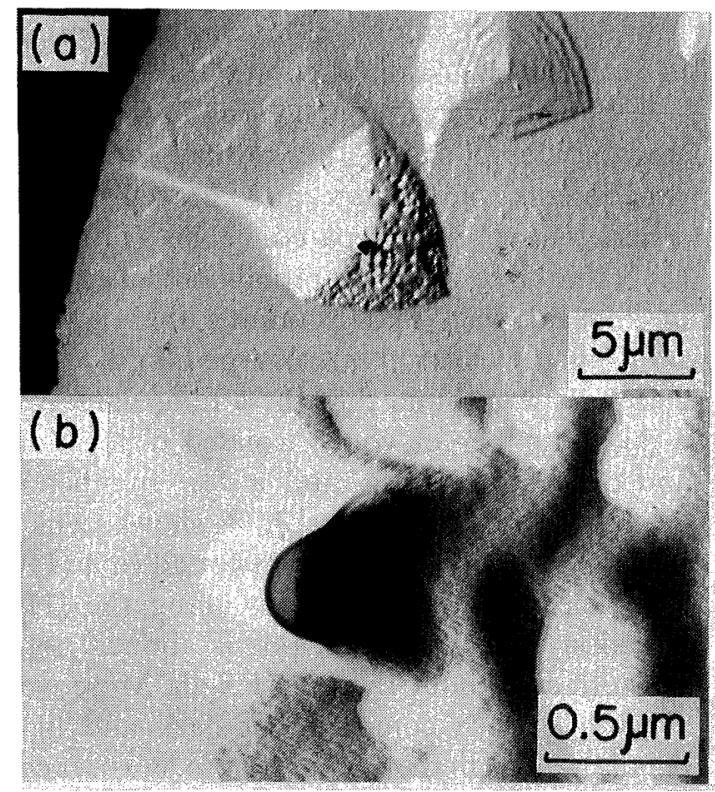

has a tendency to develop low-index facets such as (111), (110), (100) and/or their vicinal face, and consequently the beak may become flat-sided. It can also be found that the twodimensional size of anomalous pit revealed by Young's etchant is nearly the same as that revealed by Marukawa's etchant, but the former pit is much deeper than the latter one in spite of the shorter etching time. This implies that the dissolution along the defect with Young's etchant is more rapid than that with Marukawa's one. According to Marukawa ${ }^{(14)}$, dark and light etch pits revealed by his etchant on the (111) surface of copper crystals cor-

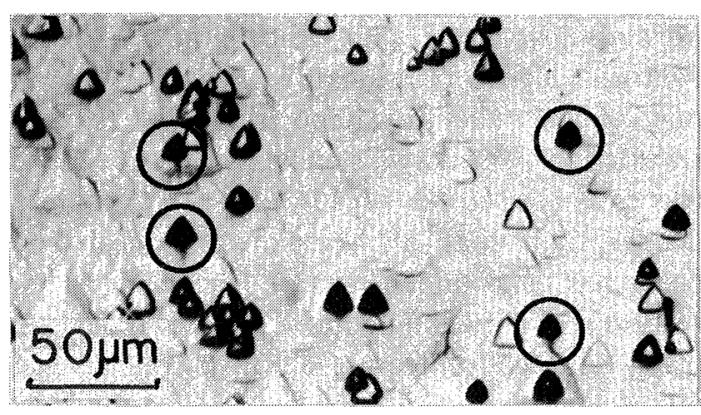

Photo. 6 Replica optical micrograph of etch pits formed on the (111) surface of a rapidly-cooled copper crystal after etched with Livingston's etchant $^{(15)}$ for $30 \mathrm{~s}$.

Photo. 7 Replica electron micrographs of an etch pit with beak formed on the (111) surface of a rapidly-cooled copper crystal after etched with Livingston's etchant $^{(15)}$ for $30 \mathrm{~s}$. (a) Dark pit with beak, (b) Enlarged top view of the etch pit with beak, (c) Enlarged top view nearly along the beak.

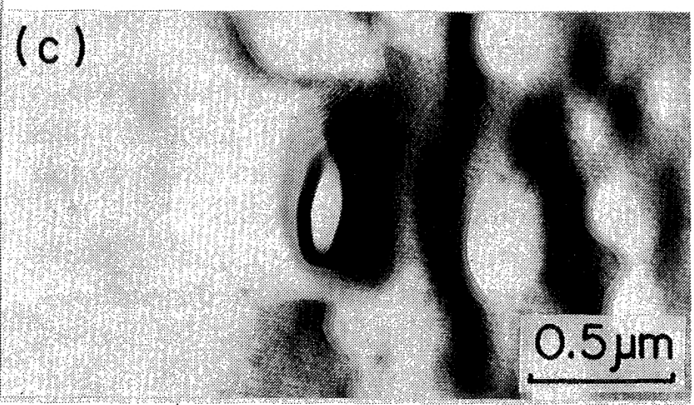


respond to screw and edge dislocations, respectively. As mentioned above, the natures of anomalous dark pits are clearly dissimilar to those of dislocation etch pits. This means that screw dislocations as well as edge ones are not responsible for the anomalous etch pits.

\section{Etching with Livingston's etchant ${ }^{(15)}$}

As already shown by Livingston ${ }^{(15)}$, dark and light pits formed by his etchant on the (111) surface of copper crystals correspond to edge dislocations with negative and positive signs, respectively. Photograph 6 shows the appearance of (111) surface of rapidly-cooled copper crystals etched with this etchant for $30 \mathrm{~s}$. Anomalous dark pits are seen in addition to normal dislocation pits. Photograph 7 is an enlarged view of anomalous pit by electron microscopy. The pit shape is similar to that revealed by Marukawa's etchant. The side slope was estimated to be $\sim 0.17 \mathrm{rad}\left(\sim 10^{\circ}\right)$, slightly deeper than that of Marukawa's ones. The beak has a rounded square shape in cross section and may extends along the [110] ([101] or [011]) direction from the bottom of the etch pit.

\section{Discussion}

It was shown that the anomalous etch pits with beaks were formed in addition to dislocation etch pits on (111) surface of rapidly-cooled copper crystals. The anomalous etch pits had several features which clearly distinguished them from others. (1) They did not appear on the (111) surface of slowly-cooled copper crystals. (2) They became larger, flat-bottomed and finally disappeared with an increase of etching time. (3) The beak extended always along an oblique $\langle 110\rangle$ ([110], [101] or [011]) direction from the bottom of the etch pit, though its shape varied with etchant.

As mentioned in $I$, the etch pits with beaks formed in some mineral and ionic crystals correspond to dislocations or tracks of fission fragments of radioactive impurities ${ }^{(4)-(11)}$. According to Hari Babu and Bansigir ${ }^{(10)}$, beaks are always associated with aged dislocations, but not with fresh dislocations in sodium chloride crystals. As suggested by them, seg- regated impurities at the aged dislocations may promote the rapid dissolution along the dislocations, presumably because the impurity atoms dissolve into etchant more easily than the normal atom, or because the impurity atoms lower the strain energy of dislocations. For the copper crystal, however, it has become apparent from the above-mentioned features that the beaked etch pits originate neither from dislocations nor from tracks of fission fragment, but instead they can be attributed to some short defects which extend into the crystal along a $\langle 110\rangle$ direction.

Barnes and Mazey ${ }^{(17)}$ performed a transmission electron microscope observation of copper (Johnson Matthey, spectroscopic purity) thin films which were annealed at $1328 \mathrm{~K}$ for $300 \mathrm{~s}$ ( $5 \mathrm{~min}$ ) in an argon atmosphere and then water quenched. Most of the films examined were found to contain a high density $(\sim 1.5 \times$ $10^{12} \mathrm{~m}^{-2}$ ) of precipitates of average radius $2 \times 10^{-8} \mathrm{~m}$, from which rows of interstitialtype dislocation loops were punched off along the $\langle 110\rangle$ directions. Since these precipitates were not observed after vacuum quenching into silicon oil, it was inferred that their formation was due to interaction with impurities in the argon atmosphere. Various secondary defects such as stacking fault tetrahedra and faulted dislocation loops were also introduced in quenched copper crystals ${ }^{(18)(19)}$. Moreover, it was known that a wide stacking fault formed in an internally oxidized copper foil ${ }^{(20)}$.

The condition of rapid cooling in the present study differed from the ordinal quenching experiments by Barnes and Mazey ${ }^{(17)}$ and others. Now, we prepared (111), (110) and (211) thin films from the rapidly-cooled crystals in a usual way, and examined them with a transmission electron microscope (JEOL, JEM200A). It was found that these films contained a large number of voids, isolated dislocations and dislocation loops, and a few number of defect ribbons. Photograph 8 shows typical patterns of the defect ribbons found in a (110) foil. The defect ribbons run along the [110] direction, and the largest one is about $0.3 \mu \mathrm{m}$ in width and about $15 \mu \mathrm{m}$ in length. Moreover, a zebra pattern composed of dark and light bands appears in the ribbon (Photo. 8(a)). It can 


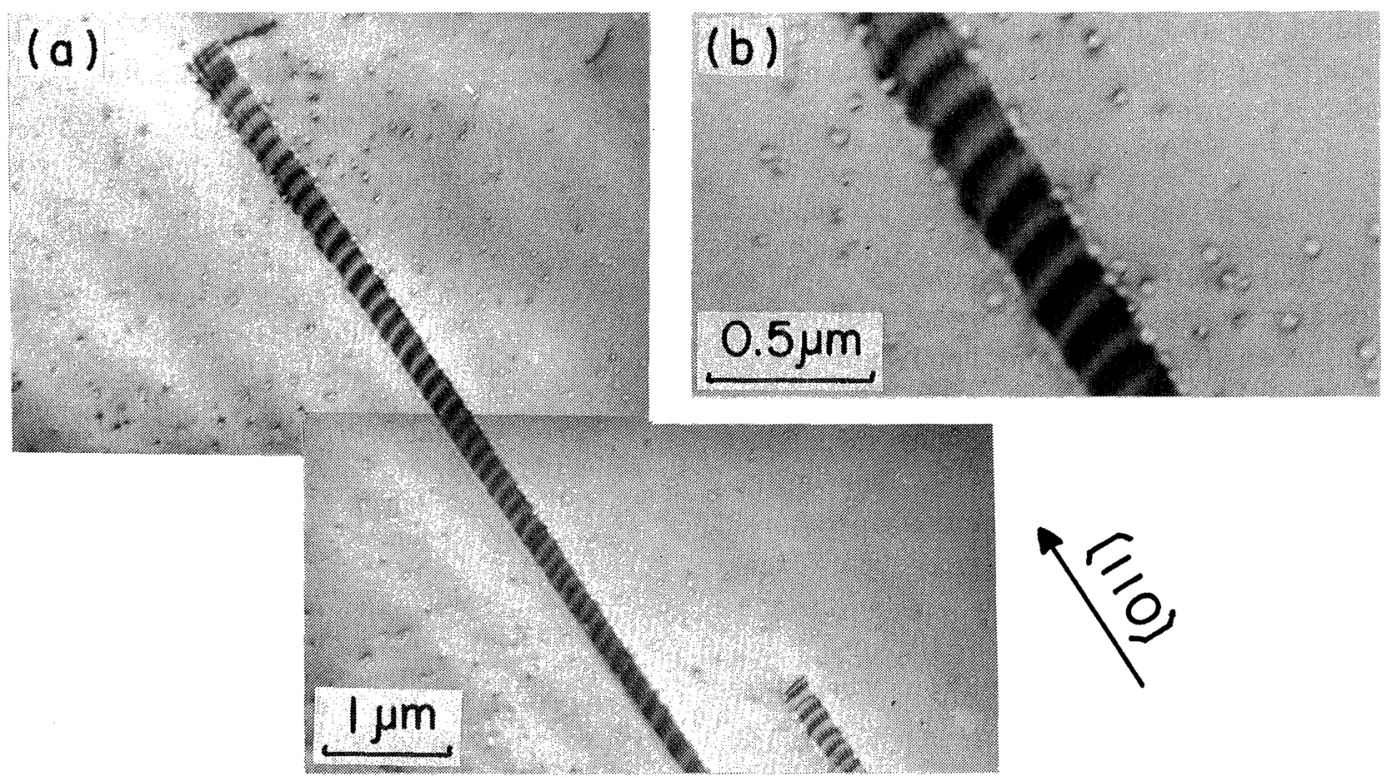

Photo. 8 Transmission electron micrographs of a (110) foil prepared from a rapidly-cooled copper crystal. (a) Defect ribbons, (b) Enlarged view.

be seen from an enlarged view (Photo. 8(b)) that the dark bands contain fringes which are generally characteristic of stacking faults, twins and thin precipitates etc. The analysis of the relations between the occurrence frequency and orientations of defect ribbons showed that they might lie on the $\{111\}$ planes. Next, some features of these defects were examined in details. Transmission electron diffraction patterns were taken at various tilting angles from a selected area containing the defect ribbons, espetially the dark bands and their neighbouring matrix. They showed only a pattern characteristic of a copper crystal, and no extra spots due to double diffraction appeared. Therefore, it seems less probable that the defect ribbons might be precipitates and/or twins. Then, bright-field and dark-field images of the defect ribbons were observed. The intensity profiles across the running directions of defect ribbons were symmetrical in the bright-field images and asymmetrical in the dark-field ones at $g=111$, $002,11 \overline{1}$ and 220 , indicating that the fringes in the dark bands might be due to stacking faults, but not to twins ${ }^{(21)}$. Then, whether the dark bands were bordered by partial dislocations or not was examined. During the observations, the dark bands in ribbons moved intermittently and the defect ribbon grew along the [110] direction. Moreover, dislocation lines could be frequently imaged at $\boldsymbol{g}=220$, but not at $\boldsymbol{g}=$ 111,002 and 111, though the partial dislocations could not be identified. A detailed observation of the light bands showed that there existed a group of parallel faint fringes intersecting them perpendicularly (Photo. 8(b)). Judging from the results of observation described above, it seems more probable that the defect ribbon might correspond to a complex row of extended dislocations between which a stacking fault was contained.

Sometimes, many voids were precipitated along both sides of defect ribbons (for example, see Photo. 8(b)), though they were not detected inside the ribbons under the condition which allowed the contrast of fringes to vanish. These voids are thought to be accumulated along the edges of defect ribbons during the rapid cooling for some reasons.

The mechanism of formation of the defect ribbons is not clear at present, and no direct evidence has been obtained that the defect ribbons are the origin of beaks. However, these defects certainly extend over a relatively short length along the $\langle 110\rangle$ directions into the crystals and they can explain as-mentioned 
features (2) and (3) of the beaked etch pits. Therefore, it is suggested that the defect ribbons may be the origin of beaks.

\section{Summary}

The (111) surfaces of copper crystals which were annealed at $1273 \mathrm{~K}$ for $7.2 \times 10^{3} \mathrm{~s}(2 \mathrm{~h})$ in the argon atmosphere and then rapidly cooled by drawing to the lower temperature part inside the furnace were etched with three kinds of dislocation etchants ${ }^{(13)-(15)}$. The optical and electron microscopic observations showed that the deep etch pits with beaks were formed with a density of $10^{7}-10^{8} \mathrm{~m}^{-2}$ in addition to dislocation etch pits of $10^{9}-10^{10} \mathrm{~m}^{-2}$. The features of the beak formation were summarized as follows:

(1) The beaked etch pits were not formed in slowly-cooled copper crystals.

(2) When the etching time increased, the beaked etch pits became gradually large and changed from sharply-pointed ones to flatbottomed ones, and simultaneously small ones newly appeared at some other places.

(3) The beaks extended along an oblique $\langle 110\rangle$ orientation from the bottom of etch pits, though the shapes of beaked etch pits varied with the etchants.

(4) In thin films obtained from the rapidlycooled crystal, the defect ribbons with a somewhat complicated structure were observed by transmission electron microscopy. It is suggested that these defects may correspond to the beaked etch pits.

\section{Acknowledgement}

The authors wish to express their thanks to Mr. K. Kikuchi for his help in the experiment.

\section{REFERENCES}

(1) A. P. Hones: The Nature, Origin and Interpretation of the Etch Figures on Crystals, John Wiley and Sons Inc., New York, (1927), p. 43.

(2) H. E. Buckley: Crystal Growth, John Wiley and Sons Inc., New York, (1951), p. 304.

(3) W. G. Johnston: Progress in Ceramic Science, Vol. II, Pergamon Press, (1961), p. 34.

(4) L. C. Lovell: Acta Met., 6 (1958), 775.

(5) R. L. Fleischer, P. B. Price and E. M. Symes: Amer. Mineral., 49 (1964), 794.

(6) A. R. Patel, M. K. Agarwal and C. C. Desai: J. Phys. Soc. Japan, 23 (1967), 553.

(7) A. R. Patel and K. K. Raju: Acta Cryst., 23 (1967), 217.

(8) A. R. C. Westwood, H. Opperhauser and D. L. Goldheim: J. Appl. Phys., 33 (1962), 1764.

(9) A. R. C. Westwood and H. Rubin: J. Appl. Phys., 33 (1962), 2001.

(10) V. Hari Babu and K. G. Bansigir: J. Appl. Phys., 40 (1969), 827.

(11) T. Hanju: J. Phys. Soc, Japan, 19 (1964), 1489.

(12) M. Yamamoto: Sci. Rep. Tôhoku Univ., 31 (1943), 121.

(13) F. W. Young, Jr.: J. Appl. Phys., 32 (1961), 192.

(14) K. Marukawa: Japanese J. Appl. Phys., 6 (1967), 944.

(15) J. D. Livingston: Direct Observation of Imperfections in Crystals, Interscience Pub., (1962), p. 115.

(16) J. Watanabé and S. Sugawara: Trans. JIM, 19 (1978), 511.

(17) B. S. Barnes and D. J. Mazey: Acta Met., 11 (1963), 281.

(18) L. M. Clarebrough, R. L. Segall and M. H. Loretto: Phil. Mag., 13 (1966), 1285.

(19) For example, S. Yoshida: Kôshikekkan to Kinzoku no Kikaiteki Seishitsu, Maruzen, (1967); p. 112 (in Japanese).

(20) P. B. Hirsch, A. Howie, R. B. Nicholson, D. W. Pashley and M. J. Whelan: Electron Microscopy of Thin Crystals, Butterworths, London, (1965), p. 61 .

(21) H. Hashimoto, A. Howie and M. J. Whelan: Proc. Roy. Soc. A, 269 (1962), 80. 